\title{
Correction to: Binary Modal Companions for Subintuitionistic Logics
}

Dick de Jongh and Fatemeh Shirmohammadzadeh Maleki

\section{Correction to:}

Chapter 2 in: M. Mojtahedi et al. (eds.), Mathematics, Logic, and their Philosophies, Logic, Epistemology, and the Unity of Science 49, https://doi.org/10.1007/978-3-030-53654-1_2

The book was inadvertently published with chapter author's incorrect family name. This information has been updated from "First Name: Dick de, Family Name: Jongh" to "First Name: Dick, Family Name: de Jongh" in the initially published version of chapter " 2 ".

The chapter and book have been updated with the change. 